\title{
Strain relaxation induced microphotoluminescence characteristics of a single InGaN-based nanopillar fabricated by focused ion beam milling
}

\author{
Peichen Yu, ${ }^{1, a)}$ C. H. Chiu, ${ }^{1}$ Yuh-Renn Wu, ${ }^{2}$ H. H. Yen, ${ }^{1}$ J. R. Chen, ${ }^{1}$ C. C. Kao, ${ }^{1}$ \\ Han-Wei Yang, ${ }^{2}$ H. C. Kuo, ${ }^{1, b)}$ T. C. Lu, ${ }^{1}$ W. Y. Yeh, ${ }^{3}$ and S. C. Wang ${ }^{1}$ \\ ${ }^{1}$ Department of Photonics and Institute of Electro-Optical Engineering, National Chiao-Tung University, \\ Hsinchu 300, Taiwan \\ ${ }^{2}$ Institute of Photonics and Optoelectronics and Department of Electrical Engineering, National Taiwan \\ University, Taipei 106, Taiwan \\ ${ }^{3}$ Electronics and Optoelectronics Research Laboratories, Industrial Technology Research Institute, \\ Hsinchu 310, Taiwan
}

(Received 21 April 2008; accepted 3 July 2008; published online 26 August 2008)

\begin{abstract}
A freestanding nanopillar with a diameter of $300 \mathrm{~nm}$ and a height of $2 \mu \mathrm{m}$ is demonstrated by focused ion beam milling. The measured microphotoluminescence $(\mu-\mathrm{PL})$ from the embedded InGaN/GaN multiple quantum wells shows a blueshift of $68 \mathrm{meV}$ in energy with a broadened full width at half maximum, $\sim 200 \mathrm{meV}$. Calculations based on the valence force field method suggest that the spatial variation of the strain tensors in the nanopillar results in the observed energy shift and spectrum broadening. Moreover, the power-dependent $\mu$-PL measurement suggests that the strain-relaxed emission region exhibits a higher radiative recombination rate than that of the strained region, indicating potential for realizing high-efficiency nanodevices in the UV/blue wavelength range. (C) 2008 American Institute of Physics. [DOI: 10.1063/1.2965461]
\end{abstract}

Recent progress in III-nitride nanofabrication technologies ${ }^{1-5}$ have opened up many possibilities for advanced optoelectronic devices in the UV/blue wavelength range, such as nanorod light emitting diode arrays, ${ }^{6}$ single nanowire lasers, ${ }^{7}$ single nanorod photodiodes, ${ }^{8}$ and nanowire sensors. ${ }^{9}$ The development of such sophisticated devices relies on the understanding of the electrical and optical properties of III-nitride nanostructures, which can be significantly altered from those of bulk. Although multiple studies have been made on the collective characteristics of GaN-based nanorods or nanowires, few reports have investigated the modified material and optical properties of a single nanostructure due to the limitations in fabrication. ${ }^{10}$ A theoretical model that describes the strain variation of $\mathrm{GaN} / \mathrm{InGaN}$ multiquantum wells (MQWs) embedded in a nanopillar had yet to be developed. In this work, a freestanding nanopillar with a diameter of $300 \mathrm{~nm}$ and a height of $2 \mu \mathrm{m}$ is fabricated using a focused ion beam (FIB). The measured microphotoluminescence ( $\mu$-PL) from the embedded $\mathrm{InGaN} / \mathrm{GaN}$ MQWs shows an energy shift of $68 \mathrm{meV}$ and a slightly broadened full width at half maximum (FWHM), which are caused by the strain relaxation of MQWs in the nanopillar. Calculations based on the valence force field (VFF) method reveal the correlation between the emission characteristics and the spatially varied polarization field. Moreover, the power-dependent $\mu$-PL measurement suggests that the strain-relaxed emission region of the nanopillar exhibits a higher radiative recombination rate than that of the strained region, making strain-relaxed InGaN-based nanopillars a promising candidate for high-efficiency UV/blue light sources.

As seen in Fig. 1(a), the epitaxial structure of the device consists of a $2 \mu \mathrm{m}$ thick GaN nucleation layer, a $2 \mu \mathrm{m}$ thick

\footnotetext{
${ }^{a)}$ Electronic mail: yup@faculty.nctu.edu.tw.

${ }^{b)}$ Electronic mail: hckuo@faculty.nctu.edu.tw.
}

Si doped $n$-GaN cladding layer, ten periods of $2.5 \mathrm{~nm}$ thick $\mathrm{In}_{0.2} \mathrm{Ga}_{0.8} \mathrm{~N}$ MQWs separated by a $10 \mathrm{~nm}$ thick $\mathrm{GaN}$ barrier, a $100 \mathrm{~nm}$ thick $\mathrm{AlGaN}$ cladding layer, and a $100 \mathrm{~nm}$ thick Mg-doped $p$-GaN layer. To prepare for the FIB milling, a $100 \mathrm{~nm}$ thick $\mathrm{SiN}_{x}$ was deposited by plasma enhanced chemical vapor deposition, followed by a $200 \mathrm{~nm}$ thick $\mathrm{Al}$ layer using electron-beam evaporation. Then, a platinum $(\mathrm{Pt})$ mask with a thickness of $500 \mathrm{~nm}$ was formed by the FIB deposition mode in an area of $500 \times 500 \mathrm{~nm}^{2}$ on the $\mathrm{Al}$ layer to provide localized protection from the ion beam sputtering. Finally, a $30 \mathrm{keV} \mathrm{Ga}$ ion beam with a current of $100 \mathrm{pA}$ was applied to remove the material around the Pt mask in a fourstep milling process. Figure 1(b) shows the field-emission scanning-electron micrograph (FESEM) of the fabricated single nanopillar. The fabricated well area was approximately $5 \times 5 \mu \mathrm{m}^{2}$, while the dimensions of the fabricated nanopillar were $\sim 300 \mathrm{~nm}$ in diameter and $\sim 2 \mu \mathrm{m}$ in height. The $\mu$-PL characteristics were investigated for the fabricated

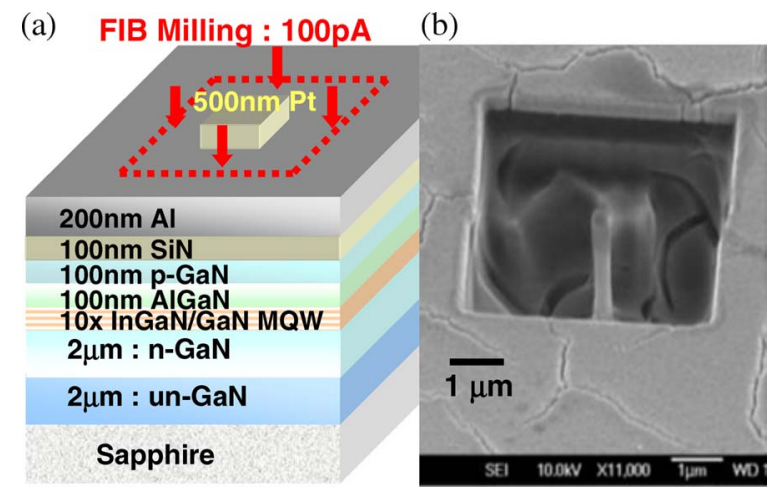

FIG. 1. (Color online) (a) Schematic of the epitaxial structure and the deposited materials for nanopillar fabrication using a focused ion beam. (b) The field-emission scanning-electron micrographic (FESEM) image of the fabricated freestanding nanopillar with a diameter of $300 \mathrm{~nm}$ and a length of $2 \mu \mathrm{m}$. 


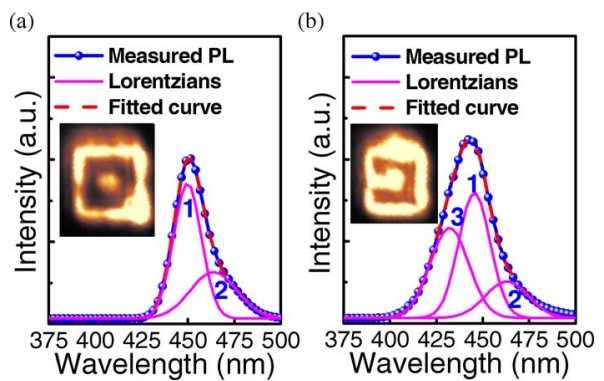

FIG. 2. (Color online) (a) The measured microphotoluminescence ( $\mu$-PL) spectrum from the freestanding nanopillar with the Pt mask and (b) without the Pt mask. The insets show the respective scanning CCD images of the device. Lorentzian analyses of the measured spectra reveal additional photoluminescence, denoted as Lorentzian 3, in the shorter wavelength region, which originates from the freestanding nanopillar.

device with and without the Pt mask at room temperature. The device was first put on a piezoelectric translator stage with a resolution of $2 \mathrm{~nm}$, where the $\mathrm{He}-\mathrm{Cd}$ laser beam with a wavelength of $325 \mathrm{~nm}$ was focused on to a spot size of $\sim 3 \mu \mathrm{m}$ by a $40 \times$ objective lens with a numerical aperture of 0.5 . The emission was then collected by the same objective lens, transmitted to a charge-coupled device (CCD) and a $0.32 \mathrm{~m}$ spectrometer, and measured at a spectral resolution of $0.1 \mathrm{~nm}$. After collecting the $\mu$-PL spectrum and the scanning image of the nanopillar with the Pt mask, the mask was removed by the metal etcher and the same structure was scanned again.

The measured $\mu$-PL spectra at an excitation power of $10 \mathrm{~mW}$ are shown in Figs. 2(a) and 2(b) for the device with and without Pt mask, respectively. The respective CCD images with a scanning area of $5 \times 5 \mu \mathrm{m}^{2}$ are displayed as the insets. As shown in the inset of Fig. 2(a), the photoluminescence from the freestanding nanopillar is weak, since the $\mathrm{Pt}$ mask blocks a significant amount of the excitation on to the nanopillar. Moreover, since the measured spectrum is very similar to that of the as-grown planar structure, we believe that the photoluminescence is mainly contributed by the emission from the periphery of the well. After the mask removal, the photoluminescence from the nanopillar becomes comparable to that from the well region, as shown in the inset of Fig. 2(b). However, the spectrum without the $\mathrm{Pt}$ mask shows additional photoluminescence in the shorter wavelength region, which is likely to originate from the freestanding nanopillar. The measured spectra are then analyzed by the Lorentzian curve fitting. As shown in Fig. 2, two and three dominant Lorentzians are found for the device with and without the mask, respectively, denoted as Lorentzians 1, 2, and 3. The peak wavelengths and the FWHMs of Lorentzians 1 and 2 in Fig. 2(a) are nearly identical to those in Fig. 2(b), confirming that both of them are photoluminescence from the well region. Therefore, it is concluded that Lorentzian 3 is the PL originating from the freestanding nanopillar. The nanopillar experiences a different strain distribution from that of the as-grown planar structure. Hence, we hereafter refer to Lorentzian 3 as the PL from the strain-relaxed region of the nanopillar and to Lorentzian 1 as that from the strained region. Lorentzian 2 is not further discussed here due to its relatively small PL intensity. The peak wavelength and the FWHM of Lorentzian 3 are 435.3 and $30 \mathrm{~nm}$, while those of Lorentzian 1 are 446 and $22 \mathrm{~nm}$, respectively. The PL from the strain-relaxed region of the nanopillar shows a blueshift
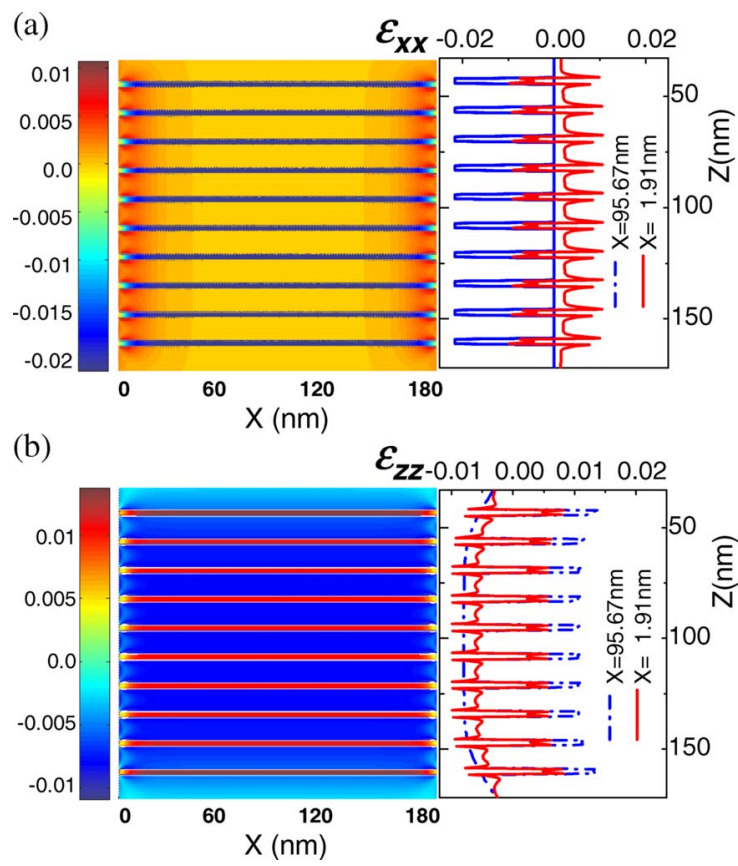

FIG. 3. (Color online) The color maps show the calculated strain variations in a freestanding, rhombus-shaped $\operatorname{In}_{0.2} \mathrm{Ga}_{0.8} \mathrm{~N} / \mathrm{GaN}$ nanopillar with a height of $Z=300 \mathrm{~nm}$ and an edge width of $X=180 \mathrm{~nm}$ for (a) in-plane strain tensor $\varepsilon_{x x}$ and (b) vertical strain tensor $\varepsilon_{z z}$. The vertical distributions of $\varepsilon_{x x}$ and $\varepsilon_{z z}$ at the center of the nanopillar, $X=95.67 \mathrm{~nm}$, and at the edge, $X=1.91 \mathrm{~nm}$, are also plotted.

of $\sim 68 \mathrm{meV}$ in energy and a FWHM slightly broadened by $\sim 72 \mathrm{meV}$, compared to those from the strained region.

Two factors that could potentially contribute to the energy shift are the quantum confined effect in the nanopillar and the strain-induced piezoelectric effect. The former cause is quickly eliminated, since the pillar diameter is too large to result in a noticeable energy shift. Moreover, it is well known that $\operatorname{In}_{x} \mathrm{Ga}_{1-x} \mathrm{~N}$ quantum wells epitaxially grown on $c$-plane $\mathrm{GaN}$ exhibit large built-in piezoelectric field due to strain, leading to band bending and the quantum-confined Stark effect. However, the strain variation of the MQWs embedded in a nanostructure can become significant, altering the distribution of the polarization field and hence the emission spectrum. It is therefore of great interest to investigate the strain variation in the nanopillar. Calculations based on the VFF model have been developed for wurtzite nanostructures. ${ }^{11}$ VFF is a microscopic model which determines the positions of each individual atom in a nanopillar by minimizing the elastic energy of the entire structure. Once the atoms' positions are known, calculating the strain tensor distribution is straightforward. To maintain the symmetry in a wurtzite structure, freestanding, rhombus-shaped GaN pillars consisting of ten-pair $\operatorname{In}_{0.2} \mathrm{Ga}_{0.8} \mathrm{~N}$ MQWs are defined with various edge widths ranging from 60 to $180 \mathrm{~nm}$. The pillars have a fixed boundary condition at the bottom and free boundary conditions on other sides. The calculated distributions of the strain tensors $\varepsilon_{x x}$ and $\varepsilon_{z z}$ are plotted in Fig. 3 for a nanopillar with a height of $Z=300 \mathrm{~nm}$ and an edge width of $X$ $=180 \mathrm{~nm}$. Both strain tensors show considerable relaxations on the outer shell of the nanopillar and as-grown-like strain distributions in the center of the nanopillar. The other inplane strain tensor, $\varepsilon_{y y}$ has a similar distribution to that of $\varepsilon_{x x}$. As shown in Fig. 3(a), the strain relaxation of $\varepsilon_{x x}$ occurs at a distance of $40 \mathrm{~nm}$ from the edge of the nanopillar. At 
(a)

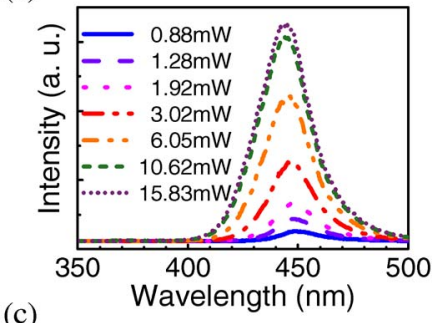

(c)

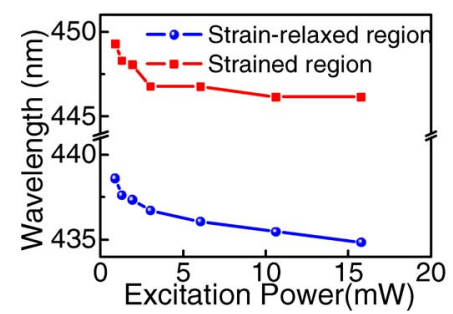

(b)

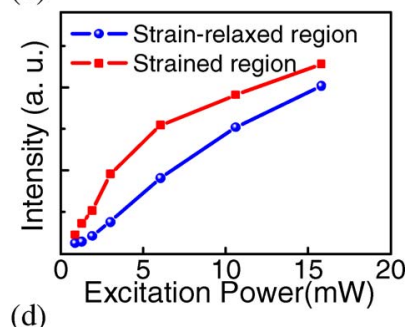

(d)

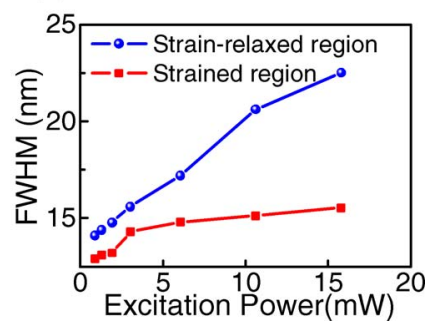

FIG. 4. (Color online) (a) The measured power-dependent $\mu$-PL spectra of the device without the Pt mask with excitation powers ranging from 0.88 to $15.83 \mathrm{~mW}$. Lorentzian analyses of the measured spectra plotted for (b) the peak intensities, (c) the emission wavelengths, and (d) the FWHMs of the PL from the strained and the strain-relaxed regions.

$X=1.91 \mathrm{~nm}$, where the change of strain from the pillar center is most significant, there exist substantial strain variations on the QW/barrier interfaces and a slight tensile strain of $\varepsilon_{x x}$ in the barriers. Moreover, as shown in Fig. 3(b), the strain relaxation of $\varepsilon_{z z}$ takes place within a distance of $\sim 10 \mathrm{~nm}$, much shorter than that of $\varepsilon_{x x}$. We also observe a vertical distribution of $\varepsilon_{z z}$ in the $Z$ direction, mainly arising from the stacking of MQWs. Both in-plane and vertical strain tensors contribute to the polarization field $P_{e z}$ via the expression

$$
P_{e z}=e_{31} \varepsilon_{x x}+e_{31} \varepsilon_{y y}+e_{33} \varepsilon_{z z},
$$

where $e_{31}$ and $e_{33}$ are piezoelectric coefficients. Based on the calculated strain shown in Fig. 3, we obtain the piezoelectric filed of $\sim 0.0276 \mathrm{C} / \mathrm{m}^{2}$ in the center of the nanopillar and of $\sim 0.01 \mathrm{C} / \mathrm{m}^{2}$ at $X=1.91 \mathrm{~nm}$ using parameters from Ref. 12 . The energy shift due to the difference in the present piezoelectric field can be estimated using a simplified structure containing an $\mathrm{In}_{0.2} \mathrm{Ga}_{0.8} \mathrm{~N} / \mathrm{GaN}$ single $\mathrm{QW}$ with a thickness of $2.5 \mathrm{~nm}$ located between a $2 \mu \mathrm{m}$ thick $n-\mathrm{GaN}$ and $100 \mathrm{~nm}$ thick $p$-GaN with doping concentrations of $10^{18} \mathrm{~cm}^{-3} \cdot{ }^{13-15}$ The carrier density $n_{2 D}$ is estimated to be $\sim 7.0 \times 10^{12} \mathrm{~cm}^{-2}$ under an excitation power of $10 \mathrm{~mW}$. An energy shift of $\sim 210 \mathrm{meV}$ is calculated, corresponding to $\lambda=415 \mathrm{~nm}$, which matches the blue edge of the PL from the strainrelaxed region, as shown in Fig. 2(b). Since the center of MQWs still suffers from strain with its magnitude comparable to that of the as-grown planar structure, the photoluminescence from the center of the pillar contributes to the rededge emission, which is also partially overlapped with the PL from the strained region, as expected. Hence, we believe that the majority of the nanopillar photoluminescence originates from the strain-relaxed region, within a distance of $\sim 40 \mathrm{~nm}$ near the edge of the nanopillar. Moreover, the variation of strain tensors in the relaxation region results in a spatially varied polarization field, leading to the broadened emission spectrum.

Figure 4(a) shows the measured power-dependent $\mu$-PL spectra of the device without the Pt mask with excitation powers varying from 0.88 to $15.83 \mathrm{~mW}$. The peak intensi-

ties, emission wavelengths, and FWHMs of the PL from the strained and the strain-relaxed regions are derived from the Lorentzian fitting of the measured spectra, as plotted in Figs. 4(b)-4(d), respectively. As seen in Fig. 4(b), the PL intensity of the strained region first increases with excitation power, and then gradually saturates for excitations larger than $6.05 \mathrm{~mW}$. The saturation can also be observed in the wavelength shift and the FWHM, as shown in Figs. 4(c) and 4(d), respectively. On the other hand, the peak intensity and the FWHM of the PL from the strain-relaxed region are monotonically increasing with the excitation. The wavelength blueshift is also more significant than that of the strained region. As the carrier density in InGaN MQWs increases with the incident power, the emission wavelength shifts toward smaller wavelengths and the spectrum becomes broader due to band filling and the screening effect of the piezoelectric field. ${ }^{16}$ However, the radiative recombination process can reduce the band filling effect in MQWs. Figures 4(b)-4(d) suggest that the transition bands are nearly filled in the strained region, where the peak intensity, wavelength shift, and the FWHM vary slowly under high excitation powers. Since the PL from the strain-relaxed region still exhibits the band filling effect under the same excitation condition as the strained region, it is deduced that the radiative recombination rate of the strain-relaxed region is faster than that of the strained region. It is also possible that other processesincluding photon extraction and non-radiative recombination-may also be involved, and further studies are necessary to completely clarify the emission mechanism. Nonetheless, we believe that strain-relaxed InGaN-based nanopillars can be a promising candidate for highly efficient UV/blue light sources.

The authors would like to thank National Science Council in Taiwan for the financial support, under Grant No. NSC96-2120-M009-006, and NSC96-2218-E-002-031 and MOEA project under Contract No. 95-EC-17-A-07-S1-011.

${ }^{1}$ H. S. Chen, D. M. Yeh, Y. C. Lu, C. Y. Chen, C. F. Huang, T. Y. Tang, C. C. Yang, C. S. Wu, and C. D. Chen, Nanotechnology 17, 1454 (2006).

${ }^{2}$ C. C. Yu, C. F. Chu, J. Y. Tsai, H. W. Huang, T. H. Hsueh, C. F. Lin, and S. C. Wang, Jpn. J. Appl. Phys., Part 1 41, 910 (2002).

${ }^{3}$ W. Q. Han, S. S. Fan, Q. Q. Li, and Y. D. Hu, Science 277, 1287 (1997).

${ }^{4}$ H. M. Kim, D. S. Kim, T. W. Kang, Y. H. Cho, and K. S. Chung, Appl. Phys. Lett. 81, 2193 (2002).

${ }^{5}$ W. Q. Han and A. Zettl, Appl. Phys. Lett. 80, 303 (2002).

${ }^{6}$ H. M. Kim, Y. H. Cho, H. Lee, S. Il. Kim, S. R. Ryu, D. Y. Kim, T. W. Kang, and K. S. Chung, Nano Lett. 4, 1059 (2004).

${ }^{7}$ J. C. Johnson, H. J. Choi, K. P. Knutsen, R. D. Schaller, P. Yang, and R. J. Saykally, Nat. Mater. 1, 106 (2002).

${ }^{8}$ M. S. Son, S. I. Im, Y. S. Park, C. M. Park, T. W. Kang and K. H. Yoo, Mater. Sci. Eng., C 26, 886 (2006).

${ }^{9}$ S. Han, W. Jin, D. Zhang, T. Tang, C. Li, X. Liu, Z. Liu, B. Lei, and C. Zhou, Chem. Phys. Lett. 389, 176 (2004).

${ }^{10}$ S. N. Yi, J. H. Na, K. H. Lee, A. F. Jarjour, R. A. Taylora, Y. S. Park, T. W. Kang, S. Kim, D. H. Ha, G. Andrew, and D. Briggs, Appl. Phys. Lett. 90, 101901 (2007).

${ }^{11}$ T. Mattila and A. Zunger, J. Appl. Phys. 85, 160 (1999).

${ }^{12}$ O. Ambacher, J. Majewski, C. Miskys, A. Link, M. Hermann, M Eickhoff, M. Stuzmann, F. Bernardini, V. Fiorentini, V. Tilak, B. Schaff, and L. F. Eastman, J. Phys.: Condens. Matter 14, 3399 (2002).

${ }^{13}$ APSYS User's Manual (Crosslight Software Inc., Burnaby, BC, 2004), available online at web page http://www.crosslight.ca

${ }^{14}$ Y. A. Chang, S. H. Yen, T. C. Wang, H. C. Kuo, Y. K. Kuo, T. C. Lu, and S. C. Wang, Semicond. Sci. Technol. 21, 598 (2006).

${ }^{15}$ F. Bernardini and V. Fiorentini, Phys. Status Solidi A 190, 65 (2002).

${ }^{16}$ J. H. Chen, Z. C. Feng, H. L. Tsai, J. R. Yang, P. Li, C. Wetzel, T. Detchprohm, and J. Nelson, Thin Solid Films 498, 123 (2006). 